\title{
Issues in Language Teaching from the Perspective of Chinese-English Language Differences
}

\author{
Bingzi Zhang,** \\ ${ }^{1}$ School of International Studies, Shaanxi Normal University, Xian, Shaanxi 710061, China \\ *Corresponding author.Email: zbz0012@foxmail.com
}

\begin{abstract}
For a long time, whenever thinking about what causes differences between languages, many scholars have defined and developed a series of definitions from the perspective of the relationship between language and thinking, so what differences exist between Chinese and English, based on language and thinking? This article will start with an understanding of the relationship between language and thinking, analyze the differences between Chinese and English in terms of language as well as the differences between Chinese and English in terms of thinking, and finally conclude how the relationship between language and thinking allows us to properly avoid and correct problems in teaching and learning.
\end{abstract}

Keywords: Languages and Cultures, differences between Chinese and Western thinking, Comparative Linguistics

\section{INTRODUCTION}

Language is a uniquely human ability, and behind the different kinds of languages in the world there are different human groups and historical cultures, all of which cannot be separated from the company of the development in human thinking. Chinese and English have distinct differences in phonetics, lexis, syntax and characters in completely different thinking conditions, and these differences can cause difficulties for language learners and teachers. Therefore, to learn or teach English or Chinese in depth, one first needs to start by understanding the relationship between language and thinking, grasping the key differences between Chinese and English, discovering the differences and similarities, and getting to know the new language in a regular and comprehensive way.

\section{LANGUAGE AND THINKING}

Language is an integral part of our lives and most people consider its existence to be as commonplace as eating and sleeping, but language carries a profound meaning. What is language? How do we define the object of language studies? What is the relationship between language and thought? From ancient times to the present, scholars from all over the world have actively explored the mysteries of language, and at the same time presented their views on it. In Modern Chinese, Huang Borong and Liao Xudong state that structurally, language is a symbolic system combining with phonetics as the material outer form, lexis as the main material, and syntax as the structural rule; functionally, it can be divided into the relationship between man and man, the relationship between man and the objective world, and the relationship between man and culture. [1] According to Chinese linguists $\mathrm{Xu}$ Tongqiang and Ye Fishing, generally speaking, the language system can be roughly divided into several subsystems such as phonetics, syntax, and lexis. [2] They also clarify that language is a symbolic system. Language, like other symbols, contains both form and meaning. For instance, the red light we see in our daily life is the form, and stopping at the red light and walking when it turns green are the meanings. A particular form expresses a particular meaning, and each particular form and meaning are inseparable.

In the language system, if words are our symbols, then phonetics is the material vehicle through which we have to emit them. Xu Tongqiang believes that the first nature of linguistic symbols is the sound that humans make. [3] Huang Borong and Liao Xudong believe that phonetics is the sound of human speech, the form of allegorical expression, or in other words, the material outer shell of language. [4] All three argue that phonetics is the material carrier of language, and that language is 
formed from the combination of speech sounds expressed in human vocal organs. Human evolution has allowed us to have an incredibly flexible vocal organs that can produce a variety of sounds by controlling the lips, teeth, jaws, throat, tongue, etc., and the air flow, and all our verbal messages are mediated by phonetics. In addition, lexis is also an important part of the language system. According to $\mathrm{Xu}$, lexis is the sum of all the words and phrases in a language (or a specific range of words). [5] Like the relationship between a person and a congregation, the words that come together are collectively known as lexis. Lexis is used throughout the oral and written expressions of a language, so the more words you have, the more you can reduce the difficulty of communication and writing. And of course, the lexis of a language can also reflect the social development behind the language, as will be discussed in more detail below. $\mathrm{Xu}$, Huang and Liao all believe that lexis is the basis for building syntax. If we compare lexis to building materials, then syntax is like a worker who knows the building process and can effectively put materials together. Syntax, as one of the elements of a language system, is the rule that shapes a language into something complete. I believe that vocabulary is the basis of the corpus of a language, phonetics is the key vehicle for oral expression, and grammar is the structural rule for combining languages, so it is important to master the three elements of a language if you want to learn it well.

In order to understand the language system, we must first clarify the object of study. In A Course in General Linguistics, the Swiss linguist Saussure makes it clear that "the common formal structure of language which exists in the mind of each individual in a linguistic community is the real object of linguistic study [6]", which means that the object of study must be, first and foremost, the people who exist in a linguistic community, and only within this community can language be acquired, for example, with wolf children raised in wolf packs which is not the case, so it is not difficult to conclude that language is a capacity unique to human societies. In addition, I do not understand the concept of linguistic communities as a whole, but rather as a division of human beings into different communities based on their mother tongue, i.e. Chinese as a mother tongue, English as a mother tongue, etc. This makes the real scope of the study clearer.

Darwin was one of the first scholars in the world to consider language as an instinct, and it can be said that language was not created abruptly, but rather evolved. We have language in the same way that birds can sing and bees can ma collect nectar. I agree with the idea that the ability to speak is an innate instinct, but what makes humans superior in the natural world is that our instincts evolve with the development of society. The Tower of Babel in the Bible describes a time when humans spoke the same language and worked together to build towers that terrified God. Having one language means that humans can communicate, collaborate and co-create. Charles F. Hockett (1958) also argues that the ability to exchange cultures allows humans to constantly stimulate each other and themselves [7] Cultural exchange is inseparable from language and also necessarily from society. For example, when we learn our mother tongue at an early age, we are able to say words such as father and mother through imitation, even though we do not know what they actually mean. The more we participate in a society, the deeper understanding of our language will be, and the more we will master it. Language, thought and society are symbiotic, and written symbols are typically a product of the symbiosis of language, thought and society. The symbols themselves do not have any natural meaning, but are given value by social convention, such as the Chinese characters with the female side, which mostly have derogatory meanings, a consequence of the patriarchal way of thinking in ancient society. Today, our society is inseparable from the internet and our thinking patterns and language development have been heavily influenced by the popularity of applications such as Weibo, where people are used to reading short and catchy sentences, thus making it progressively more difficult for people to accept longer texts.

\section{DIFFERENCES BETWEEN CHINESE AND ENGLISH}

\subsection{The Origin and History of Chinese and English}

Chinese is a very broad linguistic concept that covers dialects from all over China. And what we usually call Chinese is Mandarin. Mandarin is also known as the Common Language of the Modern Nation, which is simply a language shared by all members of one nation. Huang and Liao defined it in Modern Chinese that after studies by various parties, a three-line standard for the common language of the modern Chinese nation was formally established, namely, Beijing phonetics as the standard sound, Northern dialect as the base dialect, and exemplary modern vernacular writings as the grammatical norm. [8]" The Han Chinese are one of the oldest peoples in the world, and a common language has existed since early times. During the Spring and Autumn Period, there existed "Yayin"; during the Han Dynasty, it was collectively known as "Tongyu"; during the Ming Dynasty, it was called "Guanhua"; after the Xinhai Revolution, it was renamed " Guoyu", until the founding of New China, when the common national language was explicitly called " Mandarin ". [9] Most languages in the world exist in both spoken and written form, and Chinese, as one of the most historic languages, is no exception. When modern scholars study ancient China, it is with the help of written language texts and bronzes that can be preserved for a long time. In the changing times, some of the spoken language has died out, but his written form is 
recorded for future generations to study and research. This form of written language has to be made with the help of a linguistic symbol. Chinese characters are the only script in the world that has been in use for thousands of years without interruption or mutation. The Chinese characters, the sacred characters of ancient Egypt, the cuneiform characters of ancient Mesopotamia and the Mayan characters of the Mesoamerican region are all self-derived characters, which means that they have developed independently and have a long history of development since they were first written. But the three self-derived scripts except for Chinese characters are no longer in circulation, so it is essentially clear that Chinese can be distinguished from the other languages of the world.

Let us turn to English, one of the representatives of Germanic languages in the Indo-European system. An Introduction to Linguistics states that, as we have seen, most of the languages of Europe are considered to have a common source, a hypothetical language spoken several thousand years ago. [10] Most of the Western European languages that we see today have the same "ancestral" origin, as we described in the Biblical Tower of Babel, and English and its siblings Dutch and German all belong to the West Germanic language group. The development of English can be divided into four stages, the Old English period brought by the Anglo-Saxons, the Middle English period influenced by French, the Modern English period from the Tudor dynasty to the Restoration, and the modern English period from the late 17th century to the present day. As English has developed over its 1400-year history through the domination of different communities, the origins of the language are well documented but long obscured. Thus, English is a borrowed script.

\subsection{Textual and linguistic differences between Chinese and English}

\subsubsection{Differences in phonetic features}

Phonetics existed in both Chinese and English. The forms of phonetics we now know are the Chinese pinyin and the English phonetic alphabet, but phonetics also existed before the emergence of systematic norms for these sounds. Both Chinese and English have vowels and consonants involved, and in Chinese we commonly refer to them as "sheng mu" and "yun mu". The most essential difference is that Chinese phonetics has tones involved, and tones also play a role in discriminating meaning in Chinese.In Mandarin, for example, there are four tones: yinping, yangping, shangsheng and qusheng. Generally speaking, each word carries a tone, but sometimes a soft tone sometimes occurs when the word is formed into a word or sentence. In contrast, there is no presence of tone in English phonetics, and the most frequent occurrence in English is the 'stressed syllable', which $\mathrm{Hu}$ Zhuanglin defines that stress refers to the degree of force used in producing a syllable. [11] But in the vast majority of cases, stress does not change the meaning of the word, but only plays a role of emphasis.

\subsubsection{Differences in lexis features}

Lexis as one of the elements of a language system is a larger concept, and it can also be divided into morphemes, phrases, etc., but the minimal units of Chinese and English words are different. Hu Zhuanglin states that a morpheme can be understood as the minimal unit of language that has its own meaning [12] The term "teacher" is simply understood as a word that can be split into teach- and -er and each has its own meaning, but this form cannot be used in isolation, so in English, the morpheme is the minimal concept of lexis. In Chinese, the morpheme is what we often call a "word", but of course some people say that Chinese words can also be split into radicals, such as "Dan" (morning), which can be split into "Ri" (sun) and "Yi" (one), both of which have their own meaning, and can also be used as separate characters. This is where the difference between the smallest unit of the Chinese and English lexis lies. In An Introduction to Linguistics, it is stated that Germanic languages have a common feature that Gradation is seen in the strong verbs of the Germantic language. [13] If you want to change the tense of a verb, you only need to change the basic vowel of the word and its corresponding letter, e.g. know-knew-known; in Chinese, however, you need to add or subtract words to change the meaning, and you cannot change the meaning directly from the original word.

\subsubsection{Differences in syntax features}

There is not much difference between Chinese and English in terms of grammar. In simple terms, they are both what we call svo structures, where a sentence is made up of an action-sender subject, a predicate verb and an action-receiver object. But here I would like to mention an interesting phenomenon. When we express the occurrence of an action in the past tense and the general present tense respectively, we can see a huge difference between the two languages. In English, the present tense, the past tense and the perfect tense can be expressed by adding or subtracting letter combinations from a single verb. In Chinese, however, the meaning of each monosyllabic character is fixed and we can only add more modifiers to the sentence to achieve the purpose of expression. For example, "Wo ai ni. " and "Wo ai guo ni. " The meaning of the verb "Ai" remains the same, it is the temporal "Guo" that changes the meaning of the sentence. Sometimes the change in meaning in Chinese does not require any change in structure, but is understood in context, e.g. "Wo chi le. " There is no temporal modification, and merely the function word "Le" indicates the meaning of the past. As we mentioned above, lexis is the underlying corpus of grammar and phonetics 
is the material form of linguistic expression. In English there are clear distinctions between he, she and it in the subject, object and all-positions, as well as a greater distinction in pronunciation, but in Chinese she, he and it are all phonetically identical in the subject, object and allpositions. For example, "Wo da ta. " and "Ta da wo. " are two completely different concepts, but in English, "I hit him" and "Him hit I" have the same meaning. This makes Chinese expressions different from English, and the order of words should not be changed easily, otherwise it will cause ambiguity.

\subsubsection{Differences in character features}

Chinese characters, typically square characters, are characterised by their monosyllabic form, but there have also been two-syllable characters in history, for example, in the early nineteenth century when China translated foreign units of measurement, creating two-syllable characters, which is pronounced the same as "qian ke" $(\mathrm{kg})$. However, such characters were not widely used and even died out, so it is easy to see that monosyllabic words have always been the basic form of Chinese morphemes and have been absolutely dominant. The smallest unit in English is the morpheme, and it is difficult to use individual morphemes on their own, but requires specific combinations of morphemes.

There is a huge difference in the principles of composition between Chinese and English, and Chinese characters are also commonly known as square characters. Prior to the advent of Pinyin, form according to the meaning was the principle of character creation in Chinese, as exemplified by the oracle bone character "Li", which resembles a bean-shaped vessel filled with strings of jade, symbolising the worship of the gods with the most noble and beautiful objects. As the Chinese characters developed, they were Latinised with the introduction of Pinyin, which gradually transformed them into a script based on the principle of 'form and sound'. English is a typical sound-based script, which is made up of letters and can be read if we learn the pronunciation of each letter. This is the most fundamental difference between character of Chinese and English.

In addition to this, the composition of the soundbased script as we know it must follow a left-to-right arrangement, which is relatively homogeneous. In contrast to it, there are no specific rules for the arrangement of Chinese characters, ranging from simple forms such as left-to-right ordering, up-and-down ordering to complex and chaotic arrangements, such as the "Biang", and there are many other complex square characters like this in Chinese characters, which are completely different from sound-based characters.

\section{COMPARISON OF THE DIFFERENCES BETWEEN CHINESE AND ENGLIASH AND THE IMPACT ON LANGUAGE TEACHING}

The three main elements of language teaching are phonetics, vocabulary and grammar. Many learners are influenced by the pronunciation patterns of their mother tongue, resulting in inaccurate expressions of speech, like chinglish and Indo-English, which are often heard in English teaching. Such problems may have a negative impact on systematic learning later in life.

Chinese people generally find the learning of pronunciation the most difficult when learning English. English, as the target language of instruction, is indeed vastly different from Chinese in terms of phonetics. There are many words that begin with th- in English, such as there, this, thanks, etc. The th- morpheme is a consonant that needs to be pronounced by rubbing the tip of the tongue in the middle of both teeth, while there is no similar sound in the Chinese consonants. Also, there are many words in English that look the same, but the meaning and lexicality will change with the pronunciation, for example, the word "record" is pronounced "/'record/" as a noun and "/ri'cord/" as a verb. This adds a further obstacle to Chinese people learning and remembering word.

When learning a new language, we should not be blind to the negative effects of our mother tongue, but rather make reasonable use of our native language base. Language learning is a step-by-step process and we need to identify differences and learn and assimilate them in order to master the language. Teachers should also try to improve their own phonetic skills so that they do not mislead their students and give them the right conditions for learning the language.

\section{CONCLUSION}

Language and thinking are unique instincts that are inherent in human beings and are stimulated by social communities where language is constantly influenced by thinking and at the same time influences the development of thinking as well. Chinese and English are the two most widely spoken languages in the world, but their origins, cultural backgrounds and social groups make the two languages very different in nature. With the development of history, Chinese and English are vastly different in terms of phonetics, lexis, syntax and characters. For those teaching, a good foundation is the key to language learning, and if students are not taught the most basic elements of the language, how can we talk about getting them to think linguistically? For the learner, learning the basic elements of language is followed by a new way of thinking about that language. A deep understanding of language and thinking, and the relationship between them, 
can be of infinite help in mastering and teaching a new language.

\section{REFERENCES}

[1] B. R. Huang, X. D. Liao, Modern Chinese, Higher Education Press, Beijing, 2017, pp. 1.

[2] F. S. Ye, T. Q. Xu, Outline of Linguistics, Beijing University Press, 2010, pp. 3.

[3] F. S. Ye, T. Q. Xu, Outline of Linguistics, Beijing University Press, 2010, pp. 23.

[4] B. R. Huang, X. D. Liao, Modern Chinese, Higher Education Press, Beijing, 2017, pp. 15.

[5] F. S. Ye, T. Q. Xu, Outline of Linguistics, Beijing University Press, 2010, pp. 120.

[6] F. D. Saussure, Course in General Linguistics, The Commercial Press, 2019.

[7] S. C. Poole, An Introduction to Linguistics, Foreign Language Teaching and Research Press, Beijing, 2000, pp. 3.

[8] B. R. Huang, X. D. Liao, Modern Chinese, Higher Education Press, Beijing, 2017, pp. 3.

[9] B. R. Huang, X. D. Liao, Modern Chinese, Higher Education Press, Beijing, 2017, pp. 2.

[10] Poole, Introduction to Linguistics, Foreign Language Teaching and Research Press, Beijing, 2000, pp. 145.

[11] Z. L. Hu, A Course in Linguistics, Peking University Press, Beijing, 2017, pp. 43.

[12] Z. L. Hu, A Course in Linguistics, Peking University Press, Beijing, 2017, pp. 48.

[13] S. C. Poole, An Introduction to Linguistics, Foreign Language Teaching and Research Press, Beijing, 2000 , pp. 9. 